\title{
Teoria, pesquisa e prátic a em Educação: a formação do professor-pesquisador
}

Débora R. P. Nunes

Universidade Federal do Rio Grande do Norte

\section{Resumo}

0 presente artigo tem como objetivos pontuar fatores que contribuem para a dissociação entre teoria e prática educacional, além de enumerar as estratégias para a sua reunificação. 0 texto trata do efeito devastador dessa ruptura conceitual, que se constitui em eterno dilema entre pesquisadores, professores e gestores da área educacional. Nesse cenário, por conta da ausência de um canal de comunicação entre a Ciência e as práticas pedagógicas correntes, os resultados de pesquisa registram o seguinte: a) a ocorrência de sentimentos de ameaça à credibilidade da Educação como profissão; b) a possibilidade de os cientistas inadvertidamente delinearem modelos conceituais distanciados da realidade das salas de aula; e c) o risco de os responsáveis pela formulação de políticas públicas passarem a defender práticas educacionais que se mostram ineficazes na atualidade. Assim, 0 artigo aborda diretrizes para a reunificação dos conceitos teoria e prática educacional, destacando-se: a) a formação profissional do professor-pesquisador; b) a participação do pesquisador no cotidiano da escola; e c) o trabalho colaborativo entre o pesquisador acadêmico e o professor da sala de aula. Por fim, são apresentados dois programas educacionais norte-americanos que, com sucesso, conseguiram associar a pesquisa acadêmica à prática escolar, tendo como suporte um modelo desenvolvimentista de trabalho.

\section{Palavras-chave}

Formação de professores - Professor-pesquisador - Teoria e prática.

\footnotetext{
Correspndência:

Débora R. P. Nunes

Univ. Fed. do Rio Grande do Norte

Centro de Ciências Soc. Aplicadas

Av. Salgado Filho, $\mathrm{s} / \mathrm{n}$

59072-970 - Natal - RN

e-mail: deboranunes@ufrnet.br
} 


\title{
Theory, research and practice in Education: the formation of the teacher-researcher
}

\author{
Débora R. P. Nunes \\ Universidade Federal do Rio Grande do Norte
}

\begin{abstract}
The objective of this article is to point out factors that contribute to the dissociation between educational theory and practice, and to present the strategies for their reunification. The text deals with the devastating effect of this conceptual rift, which gives rise to an endless dilemma involving researchers, teachers, and educational managers. In such scenario, because of the lack of a communication channel between Science and current pedagogical practices, the results of this study reveal: a) the existence of feelings of threat to the credibility of Education as a profession; b) the possibility of scientists inadvertently outlining conceptual models distanced from the reality of the classrooms; and c) the risk that those responsible for public policies begin to defend educational practices that turn out to be ineffective in our days. Therefore, the article shows guidelines for the reunification of the concepts of educational theory and practice, emphasizing: a) the professional education of the teacher-researcher; b) the participation of the researcher in the school daily life; and c) the collaborative work between the academic researcher and the teacher in the classroom. Lastly, the work presents two North American educational programs that succeeded in associating academic research to school practice, using as their support a developmentalist model of work.
\end{abstract}

\section{Ke yw ords}

Teacher education - Teacher-researcher - Theory and practice.

Contact:

Débora R. P. Nunes

Univ. Fed. do Rio Grande do Norte

Centro de Ciências Soc. Aplicadas

Av. Salgado Filho, s/n

59072-970 - Natal - RN

e-mail: deboranunes@ufrnet.br 
Um dos fatores que distingue as profissões de outras ocupações é a natureza do conhecimento que se encontra subjacente à prática (Tardif, 2000). Os critérios para a elaboração e implementação de práticas profissionais em profissões como a medicina ou a engenharia são primariamente alicerçados em conhecimentos científicos especializados e formalizados e, de forma secundária, a partir do conhecimento tácito. Com o propósito de solucionar problemas concretos, esse conhecimento profissional é transformado em práticas que são modeladas e adaptadas por profissionais da área, agências governamentais, gestores, e consumidores (Greenwood; Maheady, 2001; Carnine, 2000). Diante de novas evidências empíricas, essas mesmas práticas passam a ser (re)avaliadas e, quando consideradas menos eficientes, são substituídas por práticas mais eficazes.

Esse mesmo fenômeno não é, no entanto, observado na área da Educação, na qual dados de pesquisa são regularmente ignorados, quando consideramos as práticas de sala de aula (Kauffman, 1996; Schiller; Malouf; Danielson, 1995; Greenwood; Maheady, 2001; Fuhrman, 1992; Brasil, 2003; Wideen; Mayer-Smith; Moon, 1998). Procedimentos pedagógicos ineficazes têm sido reinventados, recebido nomes populares e reintroduzidos nas escolas com o passar dos anos. Em geral, essas práticas são definidas a partir de negociações entre os profissionais da Educação, gestores e os responsáveis pela formulação de políticas públicas, desconsiderando assim o conhecimento científico (Greenwood; Maheady, 2001; Greenwood, 1998; Fuhrman, 1992).

Na década de 1970, nos Estados Unidos, os resultados de pesquisa referentes ao Projeto Follow Through ${ }^{1}$, por exemplo, comprovaram empiricamente que o método fônico de leitura produzia resultados superiores a outras oito abordagens de ensino, inclusive métodos ideovisuais de orientação construtivista. Apesar da seriedade desse estudo que envolveu 79 mil crianças provenientes de 180 comunidades nos EUA, as escolas americanas de hoje continuam utilizando procedimentos de ensino anteriormente considerados menos eficazes (Carnine, 2000).
No Brasil, os documentos e manuais didáticos oficiais - como os PCN (Parâmetros Curriculares Nacionais) (Brasil, 1998) e PNLD (Programa Nacional do Livro Didático) (Brasil, 2000) - refletem essencialmente o uso de métodos ideovisuais de leitura com orientação construtivista. As avaliações nacionais de 2003 (Brasil, 2004), no entanto, evidenciam um percentual de $55,4 \%$ de alunos que apresentam dificuldades consideráveis na atividade de leitura oral. Uma possível explicação para esse dado é que os métodos de leitura empregados pelas escolas brasileiras não são eficazes. Baseados em estudos realizados nos EUA e na Inglaterra, alguns pesquisadores no Brasil (Capovilla; Capovilla, 2003; Oliveira, 2001) argumentam que o método fônico seria mais eficaz do que os de orientação construtivista (Brasil, 2003). Devido às diferenças entre as línguas, não seria pertinente pesquisadores e educadores basearem suas conclusões e recomendações em resultados de outros países, conforme argumenta Belintane (2006). Caberia, talvez, conduzir pesquisas comparativas semelhantes ao Projeto Follow Through, considerando-se as peculiaridades das escolas brasileiras.

Os motivos do distanciamento entre a prática e a pesquisa em Educação são multivariados. Estes podem refletir desde concepções epistemológicas díspares sobre a Educação como profissão até a forma pela qual o conhecimento científico é difundido nos cursos de magistério.

A seguir, serão apontados fatores que contribuem para o distanciamento entre a prática e a pesquisa em Educação como também as alternativas para a sua reunificação.

\section{Reconceitua lizando a relação entre pesquisadores e professores}

A unificação da pesquisa científica com a prática profissional requer não somente a reconceitualização do magistério como uma

1. 0 objetivo desse projeto foi identificar métodos de ensino que seriam mais eficazes na aprendizagem de alunos da pré-escola até 3 a série do $10 \mathrm{grau}$, teve a duração de 10 anos e custou ao governo americano 600 milhões de dólares. 
profissão ancorada na própria Ciência. São necessárias também modificações nos sistemas subjacentes ao conhecimento, principalmente às relativas a desenvolvimento, validação e implementação de dados de pesquisa nas salas de aula. $\mathrm{Na}$ literatura educacional, pelo menos dois modelos direcionados para aplicação do conhecimento são proeminentes: o modelo linear e o modelo pragmático (Malouf; Schiller, 1995).

$\mathrm{Na}$ abordagem linear, modelo conceitual mais tradicional sobre a aplicação do conhecimento (Malouf; Schiller, 1995), a comunidade científica desenvolve e valida inovações educacionais e os professores aplicam-nas de forma passiva. Nessa perspectiva, os pesquisadores são percebidos como entidades que fornecem soluções prontas, visto que os professores são considerados meros consumidores "de um produto acabado". Nessa abordagem, as teorias e pesquisas vigentes determinam a maneira pela qual os problemas são estruturados. Membros da comunidade científica revalidam os métodos aplicados, e os resultados, em geral, passam a ser interpretados de forma conservadora e em referência a outros estudos e modelos compatíveis (Shavelson, 1988; Goldenberg; Gallimore, 1991). Implícito nessa abordagem encontra-se a idéia de que o saber produzido proveniente de princípios derivados de pesquisas seja a única forma de conhecimento que o professor deva possuir e aplicar.

Goldenberg e Gallimore (1991) criticam esse modelo argumentando que, embora a pesquisa científica seja capaz de identificar os principais efeitos de um procedimento, falha em considerar as interações que ocorrem quando um grande número de variáveis situacionais está envolvido. Dentre essas variáveis, figuram a história de vida do professor em ação, suas emoções, cultura e contexto em que está inserido (Tardif, 2000). Na ausência dessas informações, um "salto inferencial" é requerido na transferência de premissas científicas para premissas situacionais encontradas na prática (Shavelson, 1988). Em termos metodológicos, esse salto poderia resultar em um comprome- timento tanto da validade externa, quanto da validade social dos resultados de uma pesquisa. A validade externa refere-se à possibilidade de o pesquisador generalizar resultados obtidos a partir de ambientes artificiais para contextos reais (Wiersma, 2000). A validade social diz respeito ao grau de significância social dos resultados na perspectiva do consumidor (Wolf, 1978). Em outras palavras, é a forma como 0 professor e 0 aluno avaliam o grau de funcionalidade dos resultados da pesquisa científica, considerando sua aplicação no contexto específico de sua sala de aula.

O modelo pragmático, por outro lado, sugere que o conhecimento que emana da prática pode ser considerado funcional e válido, independentemente de pesquisas e teorias formais (Malouf; Schiller, 1995; Shulman, 1986). Esse tipo de conhecimento, a que Shulman (1986) intitula axioma do professor ou que Schön (2000) denomina conhecer-na-ação, se refere a práticas que jamais foram validadas por pesquisas empíricas, mas são utilizadas, com sucesso, por professores em sala de aula. Defensores da abordagem pragmática buscam neutralizar o predomínio da racionalidade técnica no trabalho do professor (Lüdke; Cruz, 2005) e criticam as generalizações e validações descontextualizadas dos estudos realizados em ambientes artificiais. Declaram que o conhecimento deve ser adquirido no ambiente natural, ser contextualizado e concreto (Shavelson, 1988; Goldenberg; Gallimore, 1991). No modelo pragmático, o professor é percebido como produtor e implementador de práticas educacionais que funcionam, enquanto a comunidade científica é caracterizada como produtora de estratégias que, em geral, são passíveis de aplicação apenas em ambientes rigorosamente controlados.

A centralização do trabalho na própria experiência individual e imediata do professor restringe a perspectiva de melhoria de ensino e afeta a transformação da escola em um sentido mais amplo (Lüdke; Cruz, 2005; Ramos, 2005). Em outras palavras, a supervalorização do conhecimento tácito poderia levar o profes- 
sor a ignorar as variáveis que compõem o contexto no qual ele e seus alunos estão inseridos. Em termos metodológicos, o modelo pragmático pode vir ainda a comprometer a replicação dos procedimentos utilizados pelo professor em outros contextos. Ou seja, a ausência de definição e controle das estratégias de ensino utilizadas pelo professor compromete a interpretação de seus efeitos. Em última instância, é questionado se os procedimentos adotados produziram, de fato, uma alteração no comportamento dos alunos ou se essa mudança ocorreu em função de outras variáveis.

A reconceitualização da relação entre pesquisa e prática é, nesse contexto, decisiva em Educação. É necessário ir além do modelo linear, sem ignorar o conhecimento científico. Talvez seja necessário compreender que tipos de conhecimento o professor deve adquirir e como os modelos teóricos são, de fato, aplicados nas salas de aula.

Shulman (1986) propõe um modelo no qual o conhecimento derivado da pesquisa e da prática, intitulado conhecimento proposicional, são complementados por outras fontes de conhecimento do professor. Esse autor argumenta, por exemplo, que o referencial teórico derivado da pesquisa contém informações essenciais que, apesar de simplificadas em um modelo, são de difícil aplicação em contextos específicos. Assim, Shulman (1986) introduz o conceito de conhecimento de caso, que se refere ao saber derivado de eventos específicos e minuciosamente descritos. 0 conceito envolve a descrição detalhada de como um princípio teórico ou procedimento prático pode ser utilizado em uma determinada situação, considerando as variáveis contextuais, cognitivas e afetivas. Programas de formação de professores têm, por exemplo, abordado o conhecimento de caso por meio de um procedimento didático participativo intitulado método do estudo de caso (Elksnin, 1998). Essa ferramenta de ensino favorece a análise e a crítica de casos reais provenientes do contexto escolar. Esse procedimento auxilia o aluno a compreender não apenas os axiomas do professor, mas também a funda- mentação teórica de procedimentos desenvolvidos empiricamente. Traduzir achados empíricos, considerando o conhecimento prévio e em atividades contextualizadas e familiares como nos estudos de caso, auxilia o professor a compreender a lógica de novas práticas pedagógicas (Fuhrman, 1992). Em termos práticos, a lembrança desses casos torna-se uma ferramenta fundamental no monitoramento do trabalho do professor, tanto como fonte de idéias como estímulo a novas formas de pensar (Shulman, 1986; Elksnin, 1998).

Muitas vezes, no entanto, o que é aprendido sobre um determinado princípio e o que está presente em situações reais parece incompatível. Quando isso ocorre, o professor deve desenvolver o que Shulman (1986) intitula de conhecimento estratégico, ou seja, uma consciência metacognitiva que o professor dever ter para fazer julgamentos profissionais. De fato, isso é o que diferencia o Ensino, como profissão, de um simples ofício. De acordo com Tardif (2000):

[...] o conhecimento profissional exige uma parcela de improvisação e adaptação a situações novas e únicas que exigem do profissional reflexão e discernimento para que possa não apenas compreender o problema como também organizar e esclarecer os objetivos almejados e os meios a serem usados para atingi-los. (p. 20)

Na visão de Schön (2000), isso implica em tornar-se um professor reflexivo, isto é, aquele que reflete sobre a sua ação não apenas durante o desenrolar do trabalho, mas também em seguida a seu término (Lüdke; Cruz, 2005). Na concepção de Shulman (1986), o professor torna-se um verdadeiro profissional quando é capaz de compreender, refletir, adaptar e, criticamente, programar sua ações, mesmo que isso implique em se afastar de teorias vigentes. Em uma abordagem kuhniana, esse julgamento crítico dos pressupostos teóricos em vigor gera novas formas de conhecimento e, conseqüentemente, a formulação de novas teorias. 
No modelo de Shulman (1986) e na concepção de Schön (2000), a imagem tecnicista do professor como mero consumidor ou implementador de métodos é substituída pela idéia do profissional que compreende e criticamente reflete sobre suas práticas. Esse modelo traz relevantes perspectivas sobre a forma como o conhecimento do professor deve ser investigado e estimulado. O que, no entanto, diz a literatura sobre os tipos de conhecimento que o professor utiliza em sala de aula? De que forma ele incorpora as pesquisas científicas em sua prática? Como acontece a formação do professor-pesquisador?

\section{Como o professor percebe e insere a atividade de pesquisa na escola}

A literatura sugere que os cursos de formação de professores têm um limitado impacto na prática de sala de aula (Richardson, 1994; Landrum, Cook; Tankersley, 2002; Greenwood, 1998; Gersten; Smith-J ohnson, 2001; Tardif, 2000; Lüdke; Cruz, 2005). Nesses programas instrucionais, uma série de técnicas e informações são apresentadas de forma desconexa e isolada (Gersten; Smith-J ohnson, 2001), tornando difícil a sua implementação em contextos reais. $\mathrm{Na}$ maior parte dos cursos lato sensu, por exemplo, essas informações são expostas e discutidas de forma coletiva, enquanto que a literatura tem sugerido que formas individualizadas de ensino têm apresentado resultados mais promissores na aprendizagem do professor (Langone; Koorland; Oseroff, 1987; Fuchs; Fuchs, 2001; Abbott et al., 1999). A prática exercida por meio dos estágios são, em geral, de duração insuficiente e seu conteúdo pouco relacionado ao aprendizado teórico que a precede (Lüdke; Cruz, 2005). Um outro fator agravante é o fato de disciplinas sobre metodologia e prática de pesquisa serem pouco exploradas nos cursos de magistério (Cooke et al., 1993; Lüdke; Cruz, 2005). Em estudo realizado por Lüdke e Cruz (2005), por exemplo, professores formados apontaram a falta de possibilidade de participação em programas de iniciação cien- tífica como um dos fatores determinantes da precária formação em pesquisa.

Como resultado, para muitos professores, a teoria e a pesquisa discutidas no âmbito acadêmico ocupam um papel secundário em sua formação como educadores. 0 instinto e a experiência adquiridos em sala de aula, por outro lado, passam a ser consideradas fontes principais de conhecimento (Brookhart; Freeman, 1992; Wideen; Mayer-Smith; Moon, 1998). De fato, estudantes de licenciatura tendem a enfatizar os aspectos interpessoais da profissão e considerar a experiência de campo e 0 estágio profissional como melhores recursos na formação do professor do que as aulas teóricas (Brookhart; Freeman, 1992; Wideen; Mayer-Smith; Moon, 1998). Quando esses mesmos estudantes se formam, essa visão sofre pouca ou nenhuma modificação. Pesquisas envolvendo professores da rede regular e especial de ensino mostram que esses profissionais consideram o conhecimento adquirido nos cursos de formação de professores como sendo menos fidedignos, menos relevantes e inaccessíveis do que as sugestões práticas provenientes dos colegas de trabalho (Landrum; Cook; Tankersley, 2002). Da mesma forma, diante de conflitos nas salas de aula, tendem a recorrer a modelos aprendidos na própria história familiar, escolar, e nas crenças pessoais. Em pesquisa realizada por Raymond, Butt e Yamagishi (apud Tardif, 2000), por exemplo, observou-se que, quando ocorriam problemas de disciplina em sala de aula, a tendência do professor era reativar modelos de soluções de conflitos presentes na sua própria história de vida.

Os dados de pesquisa encontrados na literatura ainda sugerem que o professor tende a priorizar estratégias de ensino condizentes com suas crenças pessoais (Wideen; Mayer-Smith; Moon, 1998). Tais crenças formam um sistema autoperpetuador que não é facilmente modificado, mesmo diante de evidências derivadas de pesquisas empíricas (Malouf; Schiller, 1995). Assim sendo, a implementação de uma nova estratégia de ensino que radicalmente difira daquilo que o professor está acostumado a fazer e que 
não seja condizente com sua história pessoal torna-se altamente improvável.

Modificar o comportamento do professor implica, portanto, que o pesquisador considere as crenças subjacentes à sua conduta (Fuhrman, 1992), assim como se familiarize com a cultura do ambiente escolar no qual esse profissional está inserido (Goldenberg; Gallimore, 1991; Fuchs; Fuchs, 2001; Malouf; Schiller, 1995). Nesse contexto, é também imprescindível que os cursos de magistério repensem suas práticas, enfatizando a importância da pesquisa científica na formação do professor. Na perspectiva de Stenhouse (apud Ramos, 2005), por exemplo, a pesquisa deveria ser a base do ensino dos professores. A formação em pesquisa é o que permite, na concepção desse autor, que o professor desenvolva uma consciência crítica de suas ações. Vale ressaltar que essa formação científica não deve ser impregnada por uma conotação unicamente acadêmica, conforme argumentam Lüdke e Cruz (2005), mas deve favorecer o trabalho voltado para questões diárias das salas de aula.

\section{A formação do professor- pesquis ador}

A literatura científica tem sugerido que professores expostos a cursos e práticas de pesquisa em programas de formação ou aperfeiçoamento de professores tendem a apresentar uma atitude mais positiva a respeito da realização de pesquisas em sala de aula (Cooke et al., 1993). De fato, o Council for Exceptional Children ${ }^{2}$ (Conselho da Criança Excepcional - Estados Unidos) inclui habilidade de pesquisa como uma competência essencial a ser desenvolvida pelo educador (Swan; Sirvis, 1992). São muitos os motivos para capacitar o professor em atividades de pesquisa no ambiente natural. Primeiramente, essa habilidade possibilitaria esse profissional a formular questões de pesquisa condizentes com o contexto da sala de aula, em vez de serem baseadas em suposições meramente teóricas. $\mathrm{Na}$ perspectiva de Shavelson (1988), isso seria essencial na sincronização da forma de pensar (mind frame) do pesquisador e do professor. Segundo, estudos conduzidos em ambientes naturais, como a sala de aula, favorecem a generalização dos resultados e aumentam a validade social da pesquisa (Wiersma, 2000). Em terceiro lugar, os professores assumiriam maior responsabilidade por suas decisões pedagógicas, uma vez que estariam capacitados a identificar os procedimentos que surtiram melhores resultados e sob quais condições (Cooke et al., 1993). Isso favoreceria o julgamento profissional do professor (Stenhouse apud Ramos, 2005) ou, conforme definido por Shulman (1986), o conhecimento estratégico, contrapondo pressupostos teóricos à prática profissional. Em quarto lugar, o professor poderia utilizar métodos experimentais na sala de aula para avaliar os efeitos de um procedimento de ensino ou fazer comparações entre diferentes práticas. Esse tipo de análise poderia levar o professor a optar por práticas pedagógicas baseadas em resultados empíricos em vez de "modismos" (Cooke et al., 1993).

Apesar dos benefícios em capacitar professores a realizarem pesquisa em sala de aula, alguns fatores tornam essa prática, por vezes, de difícil implementação. A carga de trabalho dos professores, o pouco incentivo oferecido pelos gestores, a falta de oferta de cursos de pesquisa em programas de formação de professores, a dificuldade de relacionamento entre professores e pesquisadores e a ausência de financiamento (Drew; Preator; Buchana apud Cooke et al., 1993; Lüdke; Cruz, 2005; Charlot, 2002; Ramos, 2005) são algumas razões encontradas na literatura.

\section{Associando a atividade de pesquisa à prática pedagógica}

0 gap existente entre a pesquisa educacional e a prática da sala de aula pode ser diminuído quando o professor torna-se pesquisador de sua prática e o pesquisador participante do cotidiano escolar (Fuchs; Fuchs, 2001; Charlot, 2002). A seguir serão descritos dois modelos educacio-

2. Organização profissional que descreve as competências essenciais a serem desenvolvidas pelo professor de Educação Especial. Essas competências têm servido como parâmetros para formação de professores em vários países. 
nais norte-americanos que, com sucesso, conseguiram associar a pesquisa acadêmica à prática escolar por meio de um trabalho colaborativo entre o professor e o pesquisador. Esses modelos vão ao encontro da proposta de Stenhouse (apud Ramos, 2005), na qual é sugerida a presença de pesquisadores internos (professores) e pesquisadores externos (professores universitários) no processo de construção do conhecimento.

0 primeiro modelo, intitulado Modelo de Orquestramento entre o Profissional Atuante e - Pesquisador (Practitioner and Researcher Orchestrating Model; PROMISE), foi implementado em um programa educacional cujo objetivo foi introduzir a instrução mediada por pares como estratégia de ensino em um programa de leitura (Gersten; Smith-J ohnson, 2001). O segundo modelo, denominado Projeto Infantil J upiner Gardens (J upiner Gardens Children's Project; J GCP) foi introduzido em um programa preventivo de problemas de leitura para alunos do Ensino Fundamental.

A importância do trabalho colaborativo entre o professor e o pesquisador está evidenciada na descrição de ambos os projetos. 0 primeiro módulo do J GCP, designado de componente de parceria, determina que o pesquisador permaneça entre 25 e $50 \%$ de sua jornada de trabalho no ambiente escolar onde o novo programa de ensino será implementado. o professor, por sua vez, deve formalmente concordar em colaborar com 0 projeto (Abbott et al., 1999). No modelo do PROMISE, antes de introduzir uma nova estratégia de ensino, o professor deverá analisar, juntamente com o pesquisador, o método de ensino por ele utilizado, identificando êxitos e falhas (Fuchs; Fuchs, 2001). Considerando os resultados dessa análise, o professor e o pesquisador devem, conjuntamente, definir os passos para a implementação da nova estratégia, elaborar as questões a serem investigadas, identificar o delineamento de pesquisa a ser utilizado e a forma como o novo procedimento será avaliado. Essa prática, conhecida como componente de desenvolvimento profissional, auxilia o professor a compreender importantes conceitos de pesquisa, principalmente sobre a relevância da avaliação empírica de novos procedimentos de ensino aplicados em contextos reais. Da mesma forma, o pesquisador se familiariza e aprende a incorporar o conhecimento tácito do professor em sua pesquisa.

Em seguida, os pesquisadores do J GCP tipicamente demonstram aos professores a forma como o novo procedimento deve ser gerenciado. Os professores do PROM ISE inicialmente introduzem a nova estratégia de ensino em sala de aula e, juntamente com o pesquisador, avaliam sua repercussão. Após essa avaliação, a nova estratégia poderá sofrer modificações para que se torne compatível com o estilo do professor e com a realidade da sala de aula e dos alunos. Para que os dados de pesquisa sejam incorporados à prática escolar, é necessário que sejam úteis ao professor e aos alunos (Abbott et al., 1999), ou seja, que sejam socialmente válidos (Wiersma, 2000). Isso se refere ao que o modelo J GCP intitula de componente colaborativo da intervenção, no qual pesquisadores auxiliam professores na inserção e adaptação das novas estratégias para, em última instância, favorecer a aprendizagem dos alunos.

Os efeitos da nova estratégia de ensino no comportamento dos alunos e o grau de satisfação dos professores e alunos com a introdução do novo método (validade social) são, então, mensurados no modelo PROMISE. Isso é feito por meio da instalação do programa em escolas ou salas de aula randomicamente selecionadas. Nesse processo, o pesquisador e 0 professor integrantes do modelo PROMISE oferecem cursos e workshops para que novos professores aprendam sobre o novo procedimento de ensino. Ao término do curso, esses novos professores, monitorados pelo pesquisador e professor integrante do PROMISE, implementam o novo programa de ensino em suas salas de aula. Essa etapa equivale ao que o modelo J GCP denomina fase da consultoria da intervenção.

Ao término dessa etapa, o pesquisador apresenta os dados da pesquisa aos professores que implementaram o novo método e conduz uma discussão para identificar os êxitos e as falhas encontrados. 0 grupo, como um todo, 
elabora um manual de instrução sobre o método para ser utilizado por outros professores. Consultores externos ao programa, incluindo novos professores e pesquisadores, revisam 0 material produzido. Após essa revisão, o conteúdo da pesquisa é disseminado em periódicos científicos, revistas educacionais, conferências, treinamentos em serviço e workshops.

Diferente do modelo linear previamente descrito, o PROM ISE e J GCP se fundamentam em um modelo desenvolvimentista, no qual o conhecimento científico é colaborativamente produzido. Na medida em que o professor torna-se pesquisador de sua própria prática, é desfeita a hierarquização do saber entre a universidade e a escola. Nesse modelo, o professor não mais se restringe ao papel de fornecedor de dados que vão contribuir para o trabalho de outros pesquisadores, mas está envolvido, de forma crítica, nas diversas fases da pesquisa, desde a identificação do problema até a disseminação dos resultados.

\section{C onc lusão}

A dissociação entre a pesquisa acadêmica e a prática profissional está relacionada às questões epistemológicas da Educação como profissão, aos modelos conceituais divergentes sobre a relevância do conhecimento tácito e científico, assim como às formas como a pesquisa científica é difundida nos cursos de formação de professores. A literatura tem demonstrado que as crenças e os tipos de conhecimento desenvolvidos pelo professor durante sua formação determinam, em grande parte, sua prática em sala de aula. A forma como esse professor percebe, incorpora e realiza pesquisas na escola está também relacionada a essa experiência acadêmica. A concepção tecnicista do professor como mero implementador de práticas pedagógicas ou do pesquisador como produtor de um conhecimento inaplicável precisa ser reconceitualizada. A formação profissional do professor-pesquisador favorece, em última instância, a proliferação de práticas pedagógicas eficazes. Como profissional crítico, esse professor torna-se apto a comparar métodos de ensino, refutar teorias e produzir novos conhecimentos. Alguns modelos educacionais desenvolvi-mentistas, como o PROMISE e JGCP, têm demonstrado resultados promissores no processo de unificação da teoria com a prática. 0 trabalho colaborativo entre o pesquisador acadêmico e o professor da sala de aula é apontado como um dos ingredientes fundamentais na formação do elo pesquisa- prática.

\section{Referências bibliográfic as}

ABBOTT, M. et al. Research to Practice: a "blueprint" for closing the gap in local schools. Exceptional Children, v. 65, n. 3, p. 339352, 1999. ERIC Document reproduction service. n. ED EJ 593061.

BELINTANE, C. Leitura e alfabetização no Brasil: uma busca para além da polarização. Educação e Pesquisa, São Paulo, v. 32, p. 261 277, 2006.

BRASIL. Relatório final do grupo de trabalho alfabetização infantil: os novos caminhos. In: Seminário "0 poder legislativo e a alfabetização infantil: novos caminhos" em 15 de setembro de 2003. Comissão de Cultura e Educação da Câmara dos Deputados, 2003. Disponível em <http://www.mec.gov.br>. Acesso em 02 mar. 2006.

BRASIL (SAEB). Resultados do saeb/2003. J unho de 2004 (versão preliminar). Brasilia: INEP. Disponível em <http://www.inep.gov.br/ basica/saeb/default.asp>. Acesso em 03 dez. 2006.

BRASIL. Parâmetros Curriculares Nacionais/Ensino Fundamental (1ª a 4ạ). Ministério da Educação e do Desporto. SEF. Brasília: MEC/SEF, 1998.

BRASIL. Guia do livro didático. PNLD 2000/2001. MEC, Brasília, 2000. 
BROOKHART, S.; FREEMAN, D. Characteristics of entering teacher candidates. Review of Educational Research, v. 62, p. 37-60, 1992.

CAPOVILLA, A.; CAPOVILLA, F. C. Alfabetização: método fônico. 2 ed. São Paulo, SP: Memnon; Fapesp; CNPq, 2003.

CARNINE, D. Why education experts resist effective practices (and what it would take to make education more like medicine). Washington, D.C.: Thomas B. Fordham Foundation. ERIC Reproduction Services. n. ED 442 804, 2000.

CHARLOT, B. Formação de professores: a pesquisa e a política educacional. In: PIMENTA, S. G.; GHEDIN, E. Professor reflexivo no Brasil: gênese e crítica de um conceito. 2 ed. São Paulo: Cortez, 2002. p. 89-108.

COOKE, N. et al. Teachers' opinion of research and instructional analysis in the classroom. Teacher Education and Special Education, v. 16, n. 4, p. 319-329, 1993.

ELKSNIN, L. Use of the case method of instruction in special education teacher preparation programs: a preliminary investigation. Teacher Education and Special Education, v. 21, n. 2, p. 95-108, 1998.

FUCHS, D.; FUCHS, L. One blueprint for bridging the gap: project PROMISE (practitioners and researcher orchestrating model innovations to strengthen education).Teacher Education and Special Education, v. 24, n. 4, p. 304-314, 2001.

FUHRMAN, S. Uniting producers and consumers: challenges in creating and utilizing educational research and development. In: International Seminar on Educational Research and Development. 1992. Paper. Washington, DC. ERIC Document reproduction service. n. ED 353 685, 1992.

GERSTEN, R.; SMITH-J OHNSON, J . Reflections on the research to practice gap. Teacher Education and Special Education, v. 24, n. 4, p. 356-361, 2001.

GOLDENBERG, C.; GALLIMORE, R. Local knowledge, research knowledge, and educational change: a case study of early Spanish reading improvement. Educational Researcher, v. 20, n. 8, p. 2-14, 1991.

GREENWOOD, C.; MAHEADY, L. Are future teachers aware of the gap between research and practice and what should they do? Teacher Education and Special Education, v. 24, n. 4, p. 333-347, 2001.

GREENWOOD, C. Commentary: align professional development, classroom practice, and student progress in the curriculum and you'll improve general education for all students. Learning Disabilities Quarterly, v. 21, p. 75-84, 1998.

KAUFFMAN, J. Research to practice issues. Behavioral Disorders, v. 22, n. 1, p. 55-60, 1996.

LANDRUM, T.; COOK, B.; TANKERSLEY, M. Teacher perceptions of the trustworthiness, usability and accessibility of information from different sources. Remedial and Special Education, v. 23, n. 1, p. 42-48, 2002.

LANGONE, J .; KOORLAND, M.; OSEROFF, A. Producing changes in the instructional behavior of teacher of the mentally handicapped through inservice education. Education and Treatment of Children, v. 10, n. 2, p. 146-164, 1987.

LÜDKE, M.; CRUZ, G. Aproximando universidade e escola de educação básica pela pesquisa. Cadernos de Pesquisa, v. 35, n. 125 , p. 81-109, 2005.

MALOUF, D.; SCHILLER, D. Practice and research in special education. Exceptional Children, v. 61, p. 414-424, 1995.

OLIVEIRA, J . ABC da alfabetização. Belo Horizonte: Alfaeducativa, 2002.

RAMOS, S. A pesquisa educacional inserida na formação inicial e continuada de professores: superando o distanciamento entre a universidade e a escola. Unopar Científica: Ciências Humanas e Educação. Londrina: v. 6, n. 1, p. 65-68, jun.2005 Disponível em < http://www2.unopar.br/pesq_arq/revista/HUMANAS/00000132.pdf>. Acesso em 03 jan. 2006.

RICHARDSON, V. Conducting research on practice. Educational Researcher, v. 23, n. 5, p. 5-10, 1994.

SCHILLER, E.; MALOUF, D.; DANIELSON, L. Research utilization: a federal perspective. Remedial and Special Education, v. 6, n. 6. p. 372-375, 1995. 
SCHÖN, D. Educando o profissional reflexivo: um novo design para o ensino e a aprendizagem. Porto Alegre: Artes Médicas, 2000.

SHAVELSON, R. Contributions of educational research to policy and practice: constructing, challenging, changing cognition. Educational Researcher, v. 17, n. 7, p. 4-11, 1988.

SHULMAN, R. J. Those who understand: knowledge growth in teaching. Educational Researcher, v. 15, n. 2, p. 4-14, 1986.

SWAN, W.; SIRVIS, B. The CEC common core of knowledge growth in teaching. Educational Researcher, v. 15, n.2, p. 4-14, 1986.

TARDIF, M. Saberes profissionais dos professores e conhecimentos universitários: elementos para uma epistemologia da prática profissional dos professores e suas conseqüências em relação à formação para o magistério. Revista Brasileira de Educação, Belo Horizonte, n. 13, p. 5-24, 2000.

WIDEEN, M.; MAYER-SMITH, J .; MOON, B. A critical analysis of the research on learning to teach: making the case for an ecological perspective on inquiry. Review of Educational Research, v. 68, n. 2, p. 130-178, 1998.

WIERSMA, W. Research methods in education: an Introduction. 7 ed. Boston: Allyn and Bacon, 2000.

WOLF, M. Social validity: the case for subjective measurement or how applied behavior analysis is finding its heart. J ournal of Applied Behavior Analysis, v. 11, p. 203-214, 1978.

Recebido em 19.02.07

Aprovado em 10.12.07

Débora R.P. Nunes, psicóloga, mestre em Educação pela Universidade do Estado do Rio de I aneiro e doutora em Educação Especial pela Florida State University, é professora adjunto do Departamento de Educação da Universidade Federal do Rio Grande do Norte. 